\title{
Capítulo 8 Sistema de control Mioeléctrico para silla de ruedas
}

\section{Chapter 8 Myoelectric control system for wheelchairs}

MARTÍNEZ-AGUILAR, Gloria Mónica †*, MORALES-IBARRA, Vanessa Maribel, MOTABARRAGAN, Martha y MENDIOLA-GARCIA, Yessica

Universidad Tecnológica de Torreón

ID $1^{\text {er }}$ Autor: Gloria Mónica, Martínez-Aguilar / ORC ID: 0000-0003-3834-4880, Researcher ID Thomson: G-3878-2018, CVU CONACYT ID: 213558

ID $1^{\text {er }}$ Coautor: Vanessa Maribel, Morales-Ibarra / ORC ID: 000000033764 8858, Researcher ID Thomson: F-5287-2018, CVU CONACYT ID: 387893

ID $2^{\text {do }}$ Coautor: Martha, Mota-Barragan / ORC ID: 0000-0002-7994, CVU CONACYT ID: 973093

ID $3{ }^{\text {er }}$ Coautor: Yessica, Mendiola-Garcia / ORC ID: 0000-0002-5387, CVU CONACYT ID: 599221

DOI: $10.35429 / \mathrm{H} .2019 .2 .98 .114$ 


\title{
Resumen
}

Las sillas de ruedas deben permitir que las personas con una discapacidad temporal o permanente sean incluidas en la sociedad. En el desarrollo de este trabajo, se lleva a cabo una adaptación mecánica y electrónica de un sistema de control mioeléctrico de manera simple, económica y personalizada, lo que permite al usuario manejar la silla de ruedas con facilidad, en busca de tecnologías amigables. El desarrollo de este trabajo está diseñado en cuatro etapas: detección electromiográfica, potencia, control y acoplamiento mecánico. Con el desarrollo de estas cuatro etapas, se busca simplificar de manera modular el sistema mioeléctrico a cualquier tipo de silla de ruedas manual, buscando un uso más cómodo e independiente, permitiendo adaptaciones orientadas a las necesidades de cualquier usuario.

\section{Mioeléctrico, Control, Silla de ruedas, Arduino}

\begin{abstract}
Wheelchairs must allow people with a temporary or permanent disability to be included in society. In the development of this work, a mechanical and electronic adaptation of a myoelectric control system is carried out in a simple, economical and personalized way, enabling the user to easy-handle the wheelchair, looking for friendly technologies. The development of this work is designed in four stages: electromyographic sensing, power, control and mechanical coupling. With the development of these four stages it is sought to simplify in a modular coupling way the myoelectric system to any type of manual wheelchair, looking for a more comfortable and independent use of it, permitting adaptations oriented to any user's needs.
\end{abstract}

\section{Myoelectric, Control, Wheelchair, Arduino}

\section{Introducción}

La movilidad del cuerpo humano es algo a lo que se le resta importancia, ya que diariamente la gente se desplaza de un lugar a otro para satisfacer cada una de sus necesidades y llevar a cabo acciones del quehacer cotidiano como levantarse de la cama, salir de compras o ir a pasear. Estas actividades pueden parecer cosas habituales, sin mayor dificultad o comunes y corrientes. Sin embargo, es muy importante considerar que si se tiene en casa a algún adulto mayor o familiar con alguna enfermedad que le impide movilizarse parcial o totalmente, es necesario tomar medidas al respecto para que sus movimientos vitales cotidianos sean placenteros y le permitan continuar con su vida de la forma más armoniosa posible.

En este trabajo se pretende adecuar en una silla de ruedas de uso común un sistema de control mioeléctrico, el cual además de brindar comodidad de movimiento, podrá ofrecer independencia en el usuario, sabiendo que no siempre es posible tener quién ayude al movimiento de la silla de ruedas y el hacerlo por sí mismo puede resultar, al final del día muy cansado. En la actualidad el INEGI proporciona datos que revelan que el $58.2 \%$ del total de la población con discapacidades, le corresponde a aquellos con discapacidad para caminar o moverse por sí mismos, debido a varios factores entre ellos, enfermedades, mostrando un alto porcentaje en adultos mayores.

El sistema de control propuesto está compuesto por un juego de motores, los cuales están siendo manipulados por una tarjeta Arduino uno, la cual recibe información por medio de comunicación bluetooth desde un equipo mioeléctrico, que es colocado en el antebrazo del usuario, a su vez este envía la información de los movimientos realizados. La información es procesada y posteriormente se envían los comandos pertinentes a los motores para realizar movimientos como adelante, atrás, izquierda y derecha. El sistema se acopla a la silla de ruedas de una forma sencilla y segura.

Es importante recalcar que el uso de una silla de ruedas no es exclusivo de las personas que no pueden hacer uso de sus extremidades para desplazarse. En ocasiones, para algunos adultos mayores, o personas laboralmente activas la silla de ruedas es una posibilidad de obtener mayor comodidad e interacción con su entorno social o laboral ya que les facilitará la movilidad sin desgaste físico y preocupación. 
Al realizar este proceso de conversión de la silla de ruedas es esencial recordar cuáles son las necesidades concretas que experimenta la persona, además de observar y probar la pulsera miográfíca, ya que no todas las personas tienen las mismas capacidades musculares, teniendo como ventaja que el lenguaje de programación es sencillo y fácil de adecuar para los diferentes usuarios que pudieran manejarla.

Durante el desarrollo del trabajo se presentaran las características generales de la silla de ruedas, considerando el diseño, la movilidad, los sistemas de seguridad, la ergonomía y la comodidad que sean requeridos para satisfacer las necesidades de uso, además se mostrará la programación para el manejo del control de motores, el funcionamiento de la pulsera Myo y la comunicación entre la pulsera y la tarjeta, la adecuación a la silla de ruedas, las conclusiones, mejoras y resultados obtenidos.

\section{Electromiografía}

La electromiografía de superficie (SEMG) es una técnica para la evaluación y registro de la actividad eléctrica producida por los músculos. Los músculos son órganos eléctricamente activos y las señales y patrones de señales pueden presentar información adicional sobre el estado del mismo, así como el nervio que lo suministra. Las señales generadas por electromiografía tienen una amplitud de $5 \mathrm{mV}$ y una frecuencia aproximada de 2-500 Hz.

La electromiografía es usada en aplicaciones clínicas y biomédicas como herramienta para diagnosticar enfermedades neuromusculares, y desórdenes del control motor. Las señales electromiográficas son producidas como respuesta a un movimiento muscular, donde el nivel de esfuerzo está determinado por el número de fibras musculares activadas durante la contracción por una neurona. El potencial eléctrico de una unidad motora puede ser medido utilizando electrodos de aguja o de superficie, un sistema de adquisición y procesamiento de señales mioeléctricas superficiales o SEMG se compone de diferentes etapas, iniciando por la señal mioeléctrica, pasando por etapas de amplificación, filtrado y acondicionamiento de señal para terminar en un software de análisis y poder utilizar dicha señal mioeléctrico de forma adecuada, figura 8.1.

Figura 8.1 Diagrama de bloques del sistema de adquisición de señales SEMG

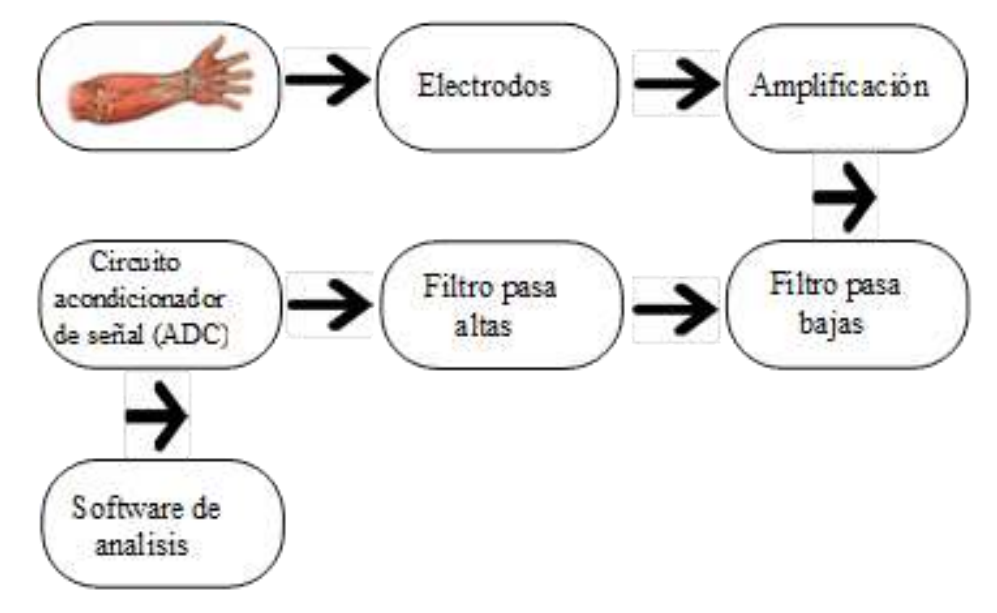

Fuente de consulta: Revista Mexicana de Ingeniería Biomédica Versión On line ISSN, 2395-9126. Sistema de detección de señales para la fatiga muscular

Las señales electromiográficas (EMG) pueden ser medidas utilizando elementos conductivos o electrodos sobre las superficie de la piel, o de manera invasiva sobre el músculo utilizando agujas. Sin embargo, la electromiografía de superficie es el método más común de medida, puesto que es no invasiva y puede ser realizada con un mínimo de riesgo sobre el paciente. La amplitud de las señales EMG varía desde los $\mu \mathrm{V}$ hasta un bajo rango de $\mathrm{mV}$ (menor de $10 \mathrm{mV}$ ).

Las señales EMG (también conocidas como MUAPs) son señales eléctricas producidas por un músculo durante el proceso de contracción y relajación. Las MUAPs son la suma de la actividad eléctrica de todas las fibras musculares que forman parte de una unidad motora (MU). La MU se compone básicamente de una neurona motora. 
La neurona motora es la encargada de emitir el impulso que provocará la contracción del conjunto de fibras, y de esta manera, provocar el movimiento del músculo.

El control mioeléctrico es un esquema de control, basado en el concepto que siempre que un músculo, en el cuerpo se contrae o se flexiona, se produce una señal eléctrica de pequeña magnitud y que es creada por la interacción química en el cuerpo.

Para este proyecto se utilizó el equipo miografico Myo Gesture Control Armband el cual adquiere la actividad mioeléctrica de los músculos del antebrazo, figura 8.2. Este equipo se detalla en el capítulo 3 .

Figura 8.2 Funcionamiento de pulsera Myo

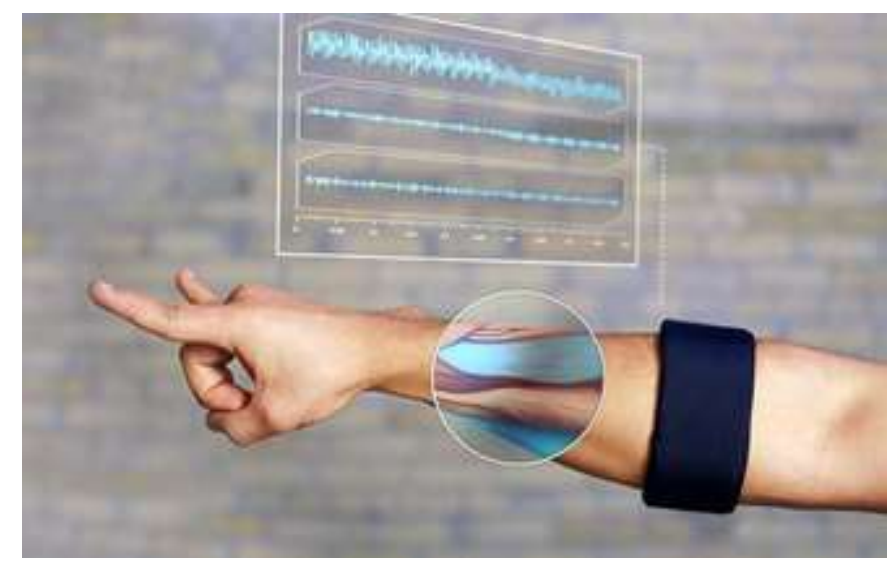

Fuente de consulta: https://www.xatakahome.com/electrodomesticos-innovadores/myo-un-brazalete-para-controlarnuestros-dispositivos-con-gestos

\section{Tipos de sillas de ruedas}

Las sillas de ruedas son dispositivos físicos de aplicación que posibilitan o mejoran la realización de actividades del aparato locomotor limitadas por deficiencias o discapacidades de tipo parcial o total, las cuales favorecen el traslado de personas que han perdido, de forma permanente, total o parcial, la capacidad de desplazarse.

En la figura 8.3 se mencionan las partes que componen una silla de ruedas, estas son importantes para el uso y manejo de la misma:

Figura 8.3 Partes de una silla de ruedas
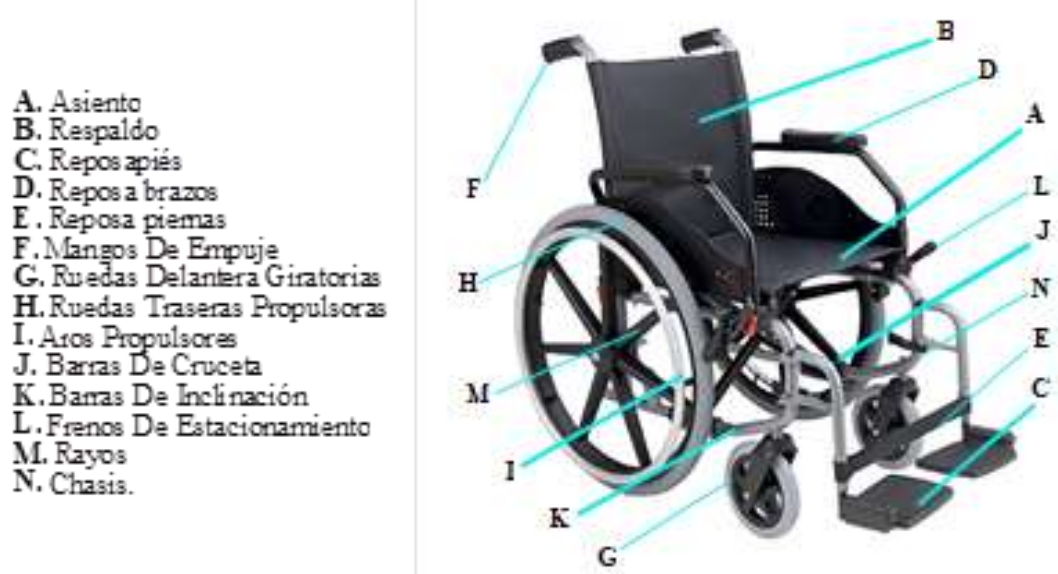

Fuente de consulta: Fuente propia 
Es importante realizar una clasificación de sillas de ruedas adecuada para el estudio y función de los dispositivos a utilizar dentro del presente trabajo, enseguida se presenta una clasificación de silla de ruedas que se divide en:

1. Sistemas dependientes: Las sillas de ruedas manuales de manejo dependiente no están diseñadas para que las controle el ocupante.

2. Sillas de transporte: Es una silla de ruedas manual controlada por un acompañante. Dispone de chasis rígido, reposapiés y reposabrazos que pueden ser abatibles y desmontables. El asiento y el respaldo suelen ser acolchados y son de material impermeable. Su función es trasladar personas con limitación para caminar mediante el manejo de la silla por un asistente.

3. Sillas de posicionamiento: Son dispositivos destinados a mantener la postura adecuada, para pacientes que tienen poca movilidad y no logran realizar los cambios de posición de forma independiente.

4. Sistemas de autopropulsión: Dispositivos diseñados para que el usuario pueda manejarlos de forma autónoma, sin necesidad de un acompañante, y cubra diversas necesidades.

5. Sillas plegables tradicionales o estándar (Ortopédicas): Son los sistemas más convencionales y de uso común para circunstancias temporales. Son plegables, ya que incorporan barras de cruceta y un asiento y un respaldo flexible de material impermeable. Esta silla se impulsa por el propio paciente manualmente.

6. Sillas Activas: A partir de información revisada, se delimita que las sillas activas son dispositivos de autopropulsión que suelen ser ultraligeros, el respaldo varía de acuerdo al nivel de equilibrio de tronco, regularmente no tienen apoyabrazos ni mangos de empuje, las ruedas traseras y el asiento suelen ser los únicos desmontables, no logran plegarse ya que cuentan con chasis rígido, aunque actualmente existen modelos nuevos que pueden compactarse y se facilita su transporte.

7. Sillas Deportivas: Varían las características de estos dispositivos de acuerdo al deporte o a las necesidades del usuario, mas tienen en común aspectos como: Chasis rígido no plegable, resistente y liviano); ruedas sumamente inclinadas que garantizan mejor movilidad giratoria, aunque aumenta el ancho total de la silla, protección en caso de choque, que también le da mayor dimensión.

8. Sistemas motorizados: Son dispositivos que permiten un fácil desplazamiento con el menor gasto energético, usada regularmente sobre terrenos regulares.

9. Sillas de ruedas eléctricas: Se conducen con un joystick. Todas las sillas de ruedas eléctricas se programan con una consola informática que permite regular la velocidad, la aceleración y la desaceleración, adaptándolas a las necesidades de cada persona.

El sistema se pensó para la automatización de sillas tradicionales en primer instancia mediante un acople mecánico fácil de implementar.

\section{Materiales y Métodos}

El diseño del sistema de control electromiográfico para sillas de ruedas se pensó de manera modular como se puede apreciar en la figura 8.4, de donde se derivan 4 etapas: etapa de sensado electromiográfico, etapa de potencia, etapa de control y etapa de acople mecánico. 
Figura 8.4 Diagrama a bloques del sistema

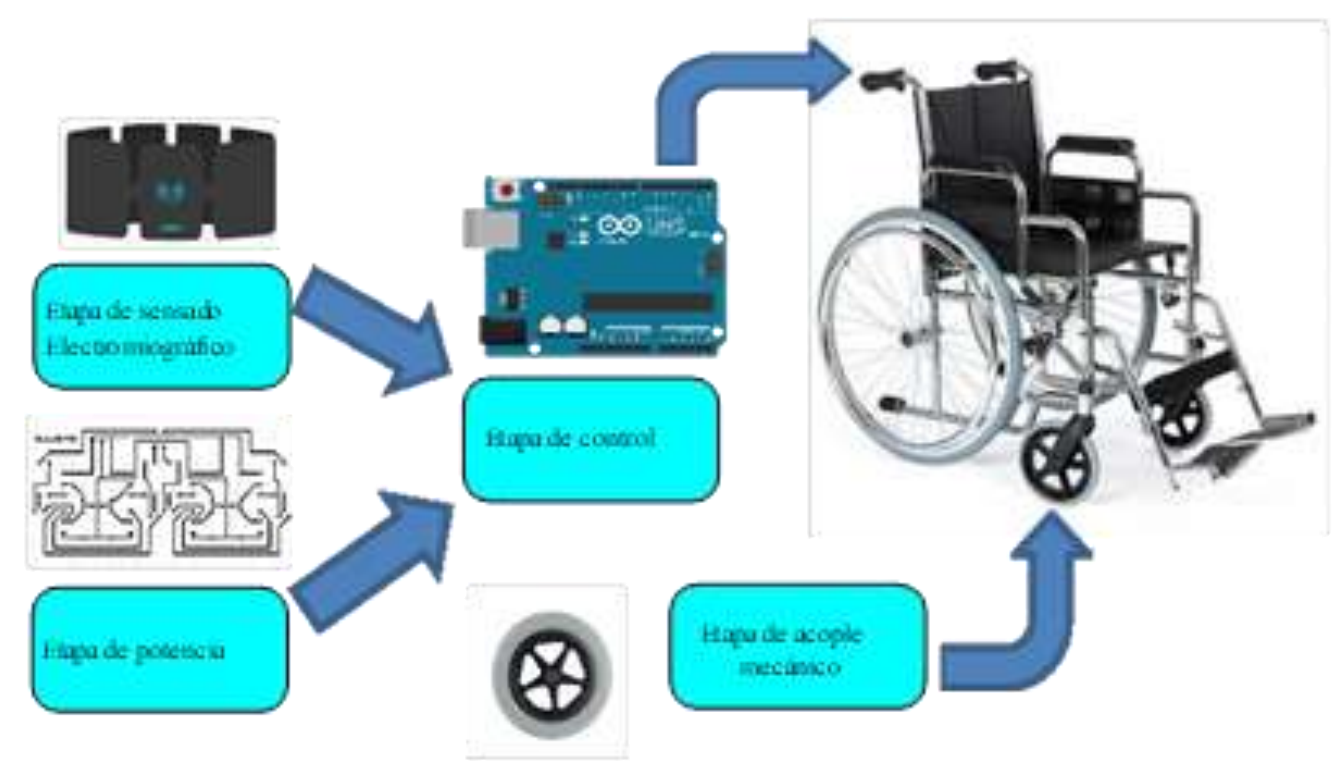

Fuente de consulta: Fuente propia

\section{Etapa de sensado electromiográfico}

En esta etapa se utilizó el equipo miografico Myo Gesture Control Armband desarrollado por la compañía Thalmic Labs, Figura 8.5, tiene ocho sensores EMG de acero inoxidable de grado médico. Al igual que otros electrodos de superficie, las señales de EMG devueltas por los sensores representan el potencial eléctrico de los músculos como resultado de la activación muscular. El rango de potenciales proporcionados por el brazalete Myo está entre -128 y 128 unidades de activación. Estas unidades de activación son valores enteros de la amplificación de los potenciales medidos por los sensores EMG. El brazalete Myo es capaz de extraer datos SEMG a una frecuencia de muestreo de $200 \mathrm{~Hz}$.

Figura 8.5 Myo Gesture Control Armband

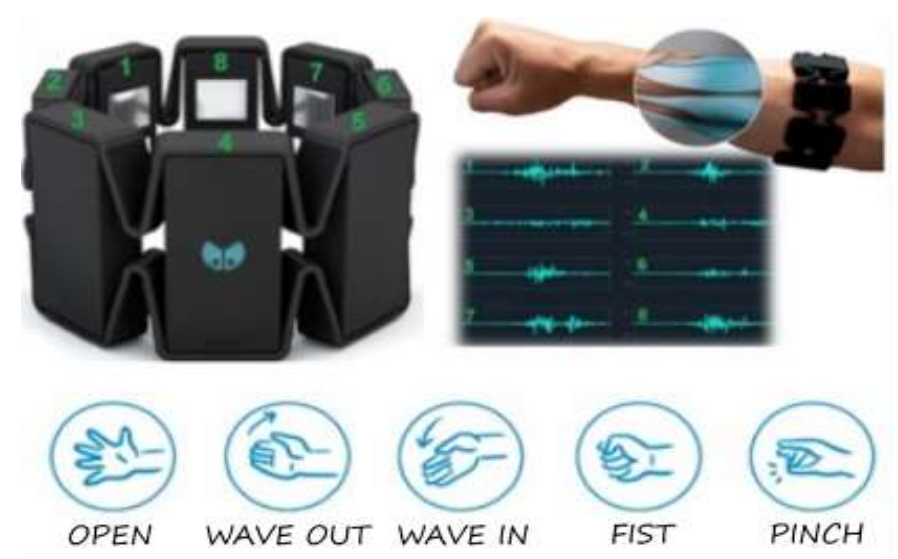

Fuente de consulta: https://www.researchgate.net/figure/Myo-armband-sensor-by-Thalmic-labs-The-bottom-imagespresent-the-possibilities-for_fig4_328676359

El brazalete Myo también tiene una unidad de medición inercial (IMU) de nueve ejes que contiene un giroscopio de tres ejes, un acelerómetro de tres ejes y un magnetómetro de tres ejes. A partir de estas unidades, la orientación y el movimiento del brazo de un usuario se pueden determinar mediante el análisis de los datos espaciales proporcionados. Los datos de orientación indican la posición del brazalete en términos de roll, pitch y yaw. La velocidad angular del brazalete se proporciona en un formato vectorial y el acelerómetro representa la aceleración que el brazalete Myo está experimentando en un momento dado. Actualmente, el brazalete Myo puede extraer datos de IMU a una frecuencia de muestreo de $50 \mathrm{~Hz}$. El brazalete Myo se ha diseñado para funcionar mejor en la parte más ancha del antebrazo, es decir, el antebrazo superior. A diferencia de otros sensores EMG, el brazalete Myo no requiere que el usuario afeite el área alrededor del cual se usará el brazalete. 

mundo real.

Esto permite procedimientos de configuración más fáciles en entornos experimentales o del

Estas características lo hacen idóneo para su aplicación en el sistema. Sin embargo, es necesario que cada usuario realice un paso de calibración antes de usar el gadget. Esto es necesario porque cada usuario tiene un tipo diferente de piel, tamaño muscular, etc., que ayudará al Myo a reconocer los gestos realizados. Otro factor importante relacionado con el enfoque de adquisición de gestos es que el Myo tiene un sensor táctil, responsable de transmitir retroalimentación (tres tipos de intervalos: corta, media y larga vibración) al usuario cuando realiza un movimiento correcto o desea activar el sistema. Para la conexión, el dispositivo utiliza la tecnología Bluetooth Low Energy, que permite una forma razonable y portable de realizar las tareas a las cuales se les implemente este sensor.

Para configurar adecuadamente la Myo es necesario seguir los siguientes pasos mencionados en el siguiente link: https://support.getmyo.com/hc/en-us/articles/203398347-Getting-started-with-yourMyo-armband

Una vez que el Myo es sincronizado y calibrado se puede conectar a la etapa de control, cabe destacar que debe conectarse a la computadora para apagarse y prenderse cada vez que se utilice. Myo Connect es una aplicación que se ejecuta en segundo plano, el ícono de la aplicación se muestra en la bandeja del sistema de Windows. Haga clic derecho para las opciones de menú. Doble toque para continuar.

Figura 8.6 Ubicación en Windows de Myo Connect

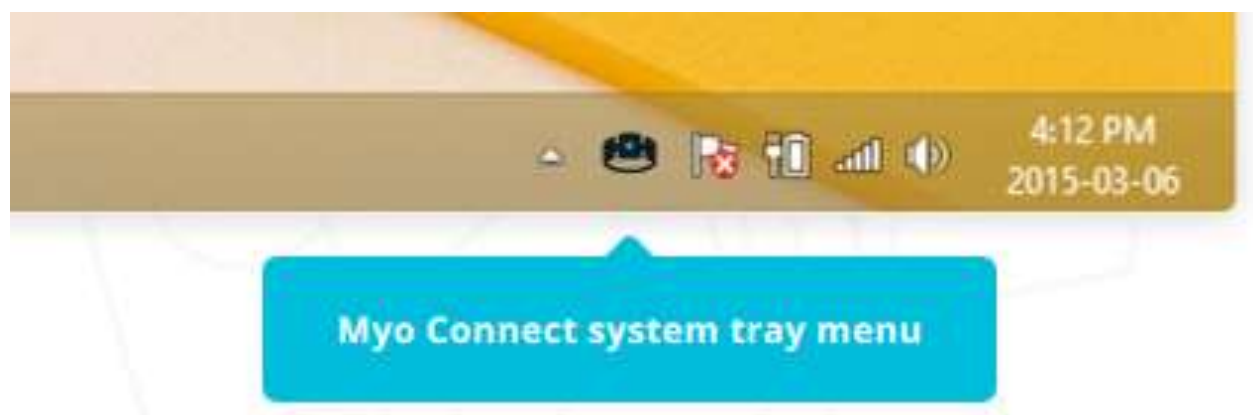

Fuente de consulta: https://support.getmyo.com/hc/en-us/articles/203398347-Getting-started-with-your-Myo-armband

Como ya se ha mencionado el equipo Myo Gesture Control Armband cuenta con reconocimiento de gestos, seguimiento de movimiento y medición de actividad muscular. Estos datos son muy útiles en varias aplicaciones, ya que los datos del sensor y los eventos se envían de forma inalámbrica a través de una conexión BLE a un dispositivo host y no se requiere un cableado complejo. Especialmente los sistemas integrados pueden beneficiarse de la portabilidad del dispositivo, como la plataforma Arduino. Desafortunadamente, la mayoría del hardware BLE tiene un conjunto de funciones muy limitado, ninguno de los módulos BLE disponibles podría conectarse directamente al Myo Armband fuera de la caja. Pero esto se resuelve mediante un firmware personalizado para estos módulos, que luego proporciona acceso a los datos de Myo y los entrega a otro dispositivo, como un Arduino. El Firmware MyoBridge se ajusta exactamente a este propósito. Es un firmware personalizado para el módulo BLE HM11.

Por lo que la conexión de la Myo a la etapa de control se realizó por medio de este módulo bluetooth 4.0, Figura 8.7, el cual es un módulo de montaje superficial (SMD) BLE usado en el BLE Bee y Xadow BLE, basado en un chip TI cc2541 que permite nodos de red robustos para que sean construidos con costos muy bajos de materiales y adecuados para sistemas de muy poco consumo de poder. El módulo es pequeño y fácil de usar, con el firmware pre programado del fabricante se pueden construir rápidamente comunicaciones BLE vía comandos AT. 
Figura 8.7 Modulo Bluetooth HM11

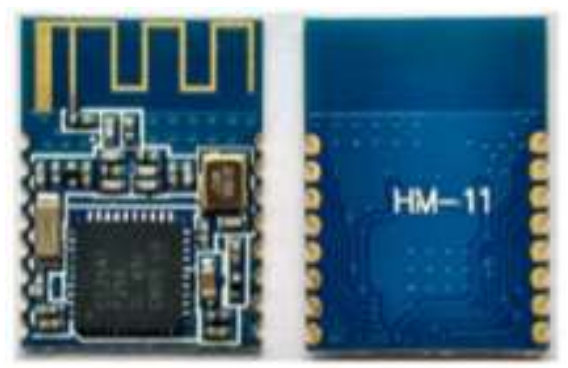

Fuente de consulta: https://www.amazon.com/Genuine-Original-Bluetooth-Wireless-Arduino/dp/B07KNX3MF5

El flasheo del Firmware MyoBridge al módulo HM11 se hace desde la tarjeta Arduino siguiendo los pasos de este link: https://blog.raquenaengineering.com/arduino-and-the-myo-armband/

Una vez terminado el flasheo, solo necesitamos probar rápidamente si todo funcionó. La carpeta MyoBridge que se descargó anteriormente viene con un par de sketchs de ejemplo, se encuentran en MyoBridge/Arduino/library/MyoBridge/examples/, pero antes de ejecutar cualquier sketch, se debe poner MyoBridge/Arduino/libraries/MyoBridge/src en la carpeta de librerias de Arduino, o comprimir la carpeta src, asígnandole el nombre MyoBridge y añadirlo al IDE de Arduino.

Después de agregar la librería se puede cargar un sketch de ejemplo y verificar la correcta comunicación con la Myo. Para esto se desconecta la Arduino una vez cargado el ejemplo y se conecta un cable desde el PIN 2 en el Arduino al PIN de TX en el Módulo BLE, y otro cable desde el PIN 3 en el Arduino al PIN de RX en el Módulo BLE. Se conecta la Arduino a la computadora, se abre el Monitor Serial y se establece la velocidad en baudios a 115200.

Si todo funciona correctamente se ve en el monitor serial la búsqueda de myo ... conectando con myo ... conectado. Seguido por la información de firmware del brazalete Myo.

Cuando el MyoBridge está conectado a la Myo, el Arduino puede enviar comandos o recibir datos de los sensores desde el Myo utilizando la librería MyoBridge.

Figura 8.8 Diagrama a bloques de conexión de Myo y Arduino

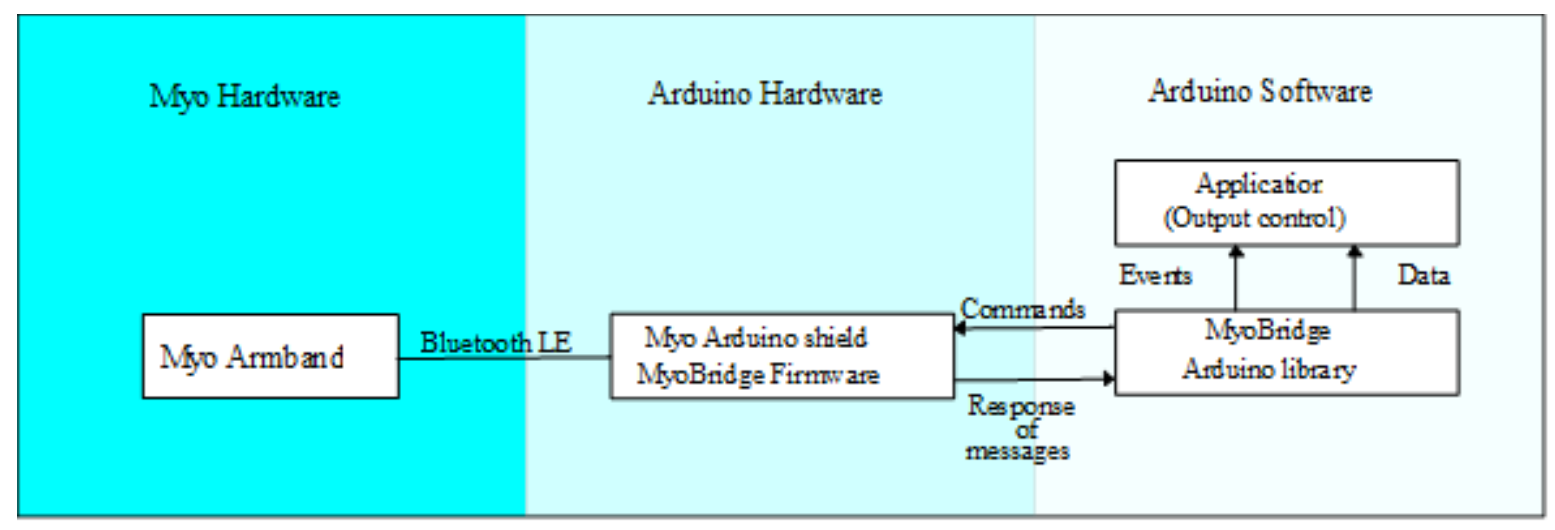

Fuente de consulta: Fuente propia

La comunicación entre HM-11 / MyoBridge y Arduino utiliza una conexión en serie simple de dos cables, mientras que el módulo HM-11 se conecta directamente al brazalete Myo. Así al hacer uso de esta librería en Arduino, se puede leer fácilmente los datos del Myo, sin necesidad de una PC o teléfono intermedio. Los datos se pueden adquirir de tres maneras:

a. EMGData.- Obtienen los datos raw de los 8 sensores SEMG.

b. IMUData.- Obtienen los datos de la IMU.

c. PoseData.- Obtienen en conjunto las anteriores e interpreta la seña realizada. 
En el caso del control propuesto se utiliza el modo PoseData. Este modo reconoce cinco movimientos más el descanso del brazo.

Figura 8.9 Movimientos reconocidos por la MyoBridge

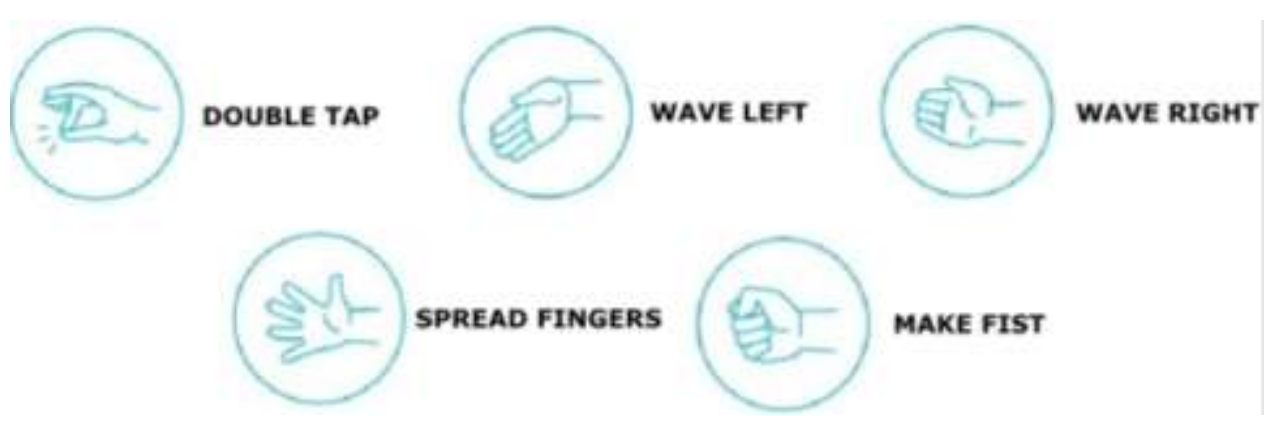

Fuente de consulta: Fuente propia

\section{Etapa de potencia}

La etapa de potencia se realizó para el debido control de velocidad y giro con los actuadores del sistema los cuales son dos motores eléctricos elevalunas de $12 \mathrm{~V}$ a $7 \mathrm{~A}$. Para esto se diseñó un puente $\mathrm{H}$.

Figura 8.10 Diagrama eléctrico de un puente $\mathrm{H}$ con transistores de potencia

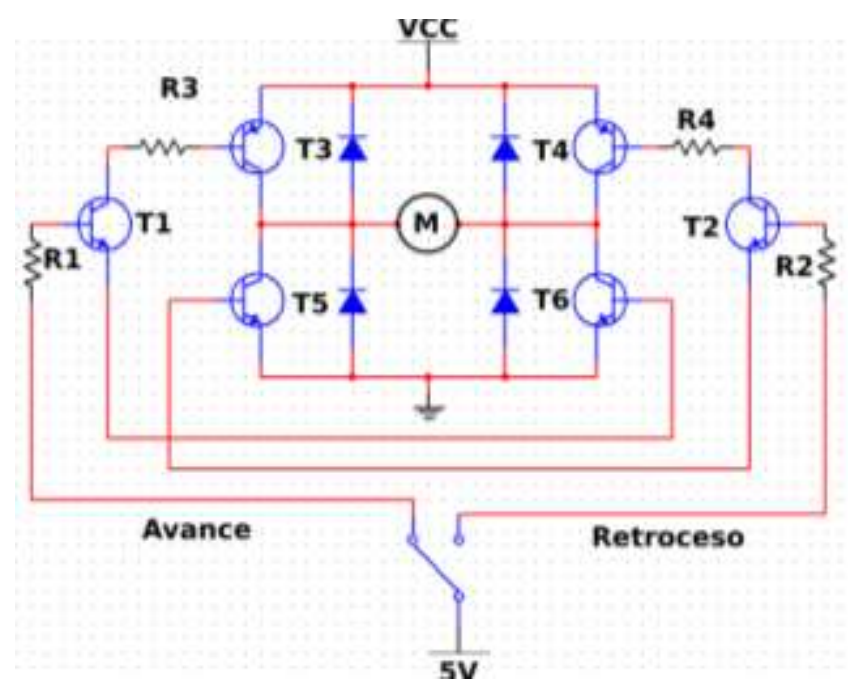

Fuente de consulta: Fuente propia

Este se implementó con elementos de potencia para soportar la corriente necesaria en los motores. El diseño del PCB se realizó en PCB Wizard como se muestra en la Figura 8.11, la implementación del circuito se elaboró mediante el método de planchado, para su posterior soldadura de los elementos dando como resultado la Figura 8.12.

Figura 8.11 Diseño PCB de puente $\mathrm{H}$

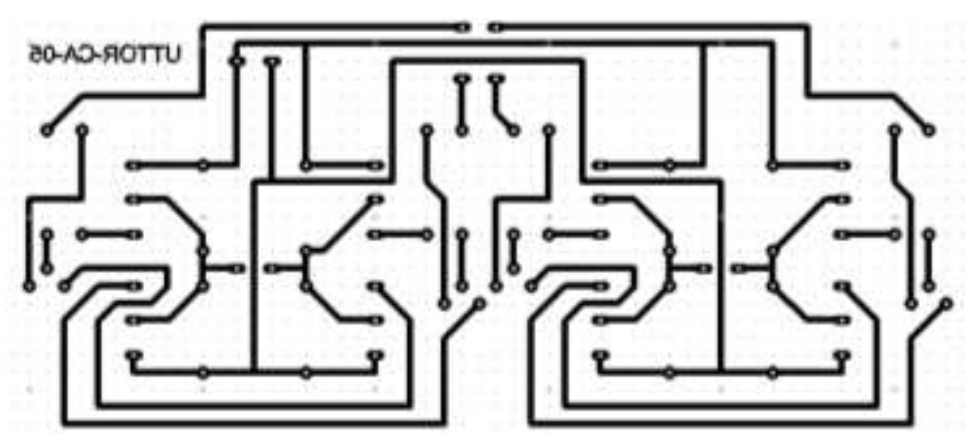

Fuente de consulta: Fuente propia 
Figura 8.12 Puente H elaborado. (a) parte inferior y (b) parte superior

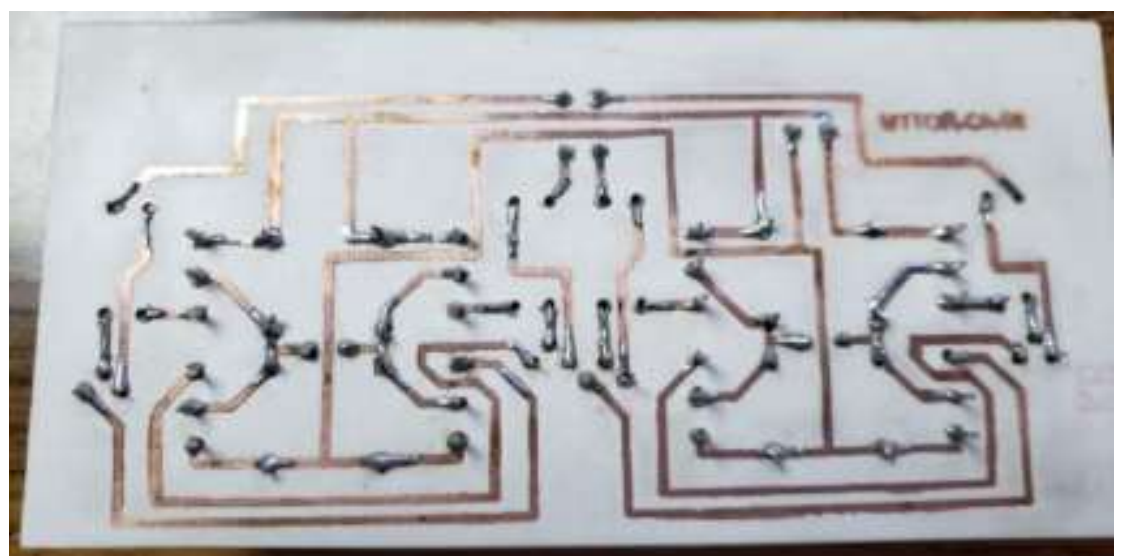

(a)

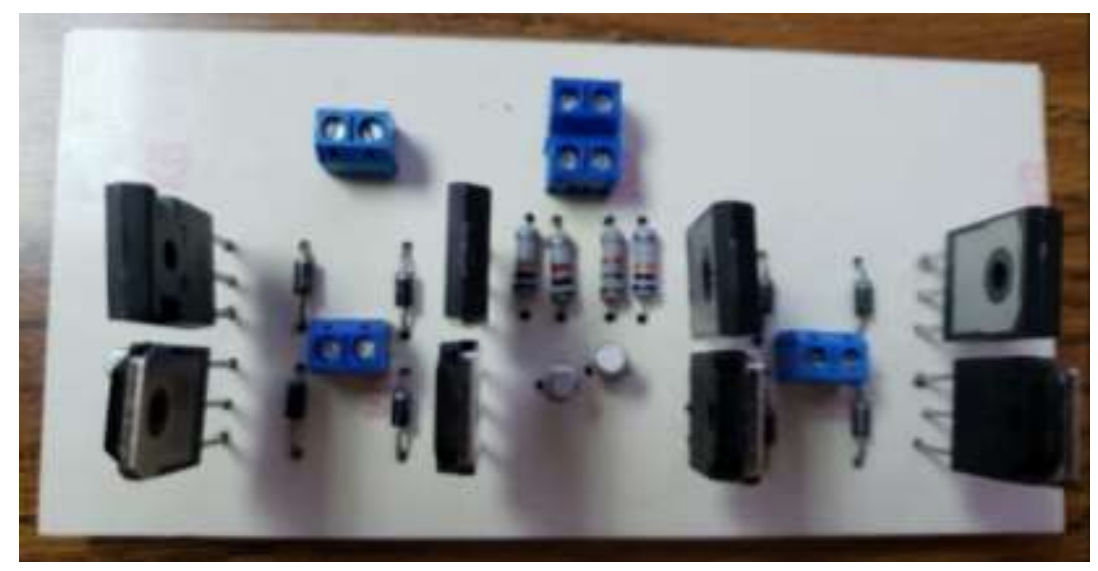

(b)

Fuente de consulta: Fuente propia

Después de la elaboración del puente $\mathrm{H}$, para la etapa de control se define la tabla de verdad de los direccionamientos que realizarán cada uno de los motores. La definición de esos movimientos es esencial para el posterior enclavamiento de estos con los gestos capturados con la Myo. Como se puede observar en la Figura 8.10 se necesita una combinación de dos bits para el control del puente $\mathrm{H}$ lo cual da como resultado una combinación de cuatro posibles direccionamientos, en este caso son dos puentes $\mathrm{H}$, por lo tanto son cuatro bits con un total de 16 posibles direcciones, de las cuales no todas son factibles o de utilidad en el sistema propuesto. En la tabla 8.1 se definen las combinaciones que resultan en direcciones útiles y las que no serán tomadas en cuenta. Donde el Motor 1 se toma como el de la izquierda y el Motor 2 como el de la derecha. En cuanto a las velocidades de los motores, éstas son definidas mediante la etapa de control con un modulador de ancho de pulsos (también conocida como PWM, por sus siglas en inglés de pulse-width modulation), la cual es una técnica en la que se modifica el ciclo de trabajo de una señal periódica (una senoidal o una cuadrada, por ejemplo), ya sea para transmitir información a través de un canal de comunicaciones o para controlar la cantidad de energía que se envía a una carga. El ciclo de trabajo de una señal periódica es el ancho relativo de su parte positiva en relación con el período. Expresado matemáticamente:

$$
D=\frac{\tau}{T}
$$

Donde:

D es el ciclo de trabajo

$\tau$ es el tiempo en que la función es positiva (ancho del pulso)

$\mathrm{T}$ es el período de la función

En nuestro caso se manejan tres velocidades relativas al PWM: baja (30\% de ciclo de trabajo), media ( $50 \%$ de ciclo de trabajo) y alta (90\% de ciclo de trabajo). 
Tabla 8.1 Combinaciones de direccionamiento para puente $\mathrm{H}$

\begin{tabular}{|l|l|l|l|l|}
\hline \multicolumn{2}{|c|}{ Motor 1 } & \multicolumn{2}{c|}{ Motor 2 } & \multicolumn{1}{c|}{ Direccionamiento } \\
A1 & B1 & A2 & B2 & \\
\hline 0 & 0 & 0 & 0 & Stop \\
\hline 0 & 0 & 0 & 1 & No valido \\
\hline 0 & 0 & 1 & 0 & No valido \\
\hline 0 & 0 & 1 & 1 & Stop \\
\hline 0 & 1 & 0 & 0 & No valido \\
\hline 0 & 1 & 0 & 1 & Derecha \\
\hline 0 & 1 & 1 & 0 & No valido \\
\hline 0 & 1 & 1 & 1 & No valido \\
\hline 1 & 0 & 0 & 0 & No valido \\
\hline 1 & 0 & 0 & 1 & No valido \\
\hline 1 & 0 & 1 & 0 & Izquierda \\
\hline 1 & 0 & 1 & 1 & No valido \\
\hline 1 & 1 & 0 & 0 & Stop \\
\hline 1 & 1 & 0 & 1 & No valido \\
\hline 1 & 1 & 1 & 0 & No valido \\
\hline 1 & 1 & 1 & 1 & Stop \\
\hline
\end{tabular}

Fuente de consulta: Fuente propia

Como se puede apreciar en la tabla 8.1 hay muchas direcciones No validas o Stop, esto es debido que para que el puente $\mathrm{H}$ funcione debe tener sus combinaciones diferentes, si son iguales el motor se queda enclavado, por lo mismo ambos motores deben de moverse para generar una vuelta.

\section{Etapa de control}

Como ya se ha venido mencionando la etapa de control es la parte principal del sistema propuesto, es donde se adquieren las señales de entrada del equipo Myo se procesan y se envían los datos de direccionamiento y velocidad a la etapa de potencia. Esta etapa se encuentra embebida en una tarjeta Arduino uno R3.

Arduino Uno es una placa electrónica basada en el microcontrolador ATmega328. Cuenta con 14 entradas/salidas digitales, de las cuales 6 se pueden utilizar como salidas PWM y otras 6 son entradas analógicas. Además, incluye un resonador cerámico de $16 \mathrm{MHz}$, un conector USB, un conector de alimentación, una cabecera ICSP y un botón de reseteado. La placa incluye todo lo necesario para que el microcontrolador haga su trabajo, basta conectarla a un ordenador con un cable USB o a la corriente eléctrica a través de un transformador.

Figura 8.13 Esquemático de tarjeta Arduino Uno R3

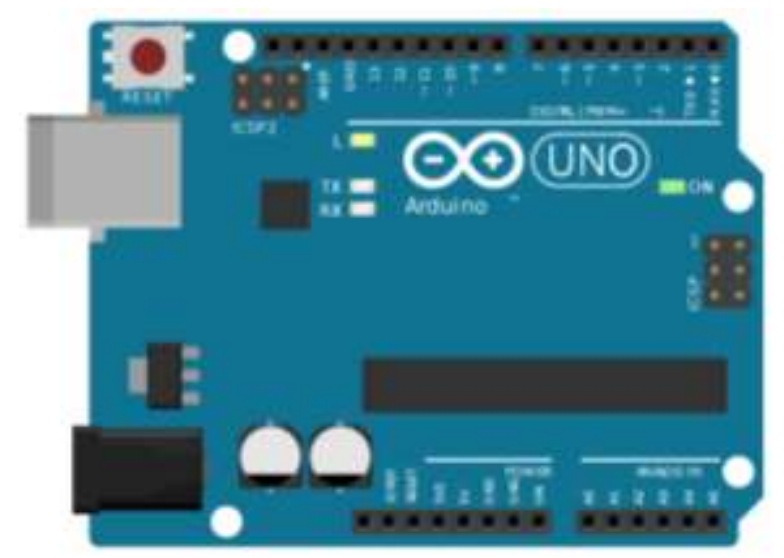

Fuente de consulta: https://commons.wikimedia.org/wiki/File:ArduinoUno.svg

El esquema general de conexión del Arduino en el sistema propuesto se puede observar en la Figura 8.14, donde anteriormente ya se había comentado la conexión del módulo HM11 a los pines digitales 2 (verde) y 3 (azul), el modulo también se alimenta de la tarjeta Arduino (rojo VCC y negro GND). 
La etapa de potencia se conecta a dos pares de los pines PWM, pines 6 y 7 para el motor 2 (cables rojo claro) y pines 9 y 10 para el motor 1 (cables rojo oscuro); a su vez para la alimentación de los motores se conecta a una batería de $12 \mathrm{~V}$ a $7 \mathrm{~A}$ (voltaje positivo cable morado) donde la tierra (cable negro) es la misma para todos los elementos para evitar voltajes diferenciados en el sistema.

Figura 8.14 Esquema general de conexión de etapa de control

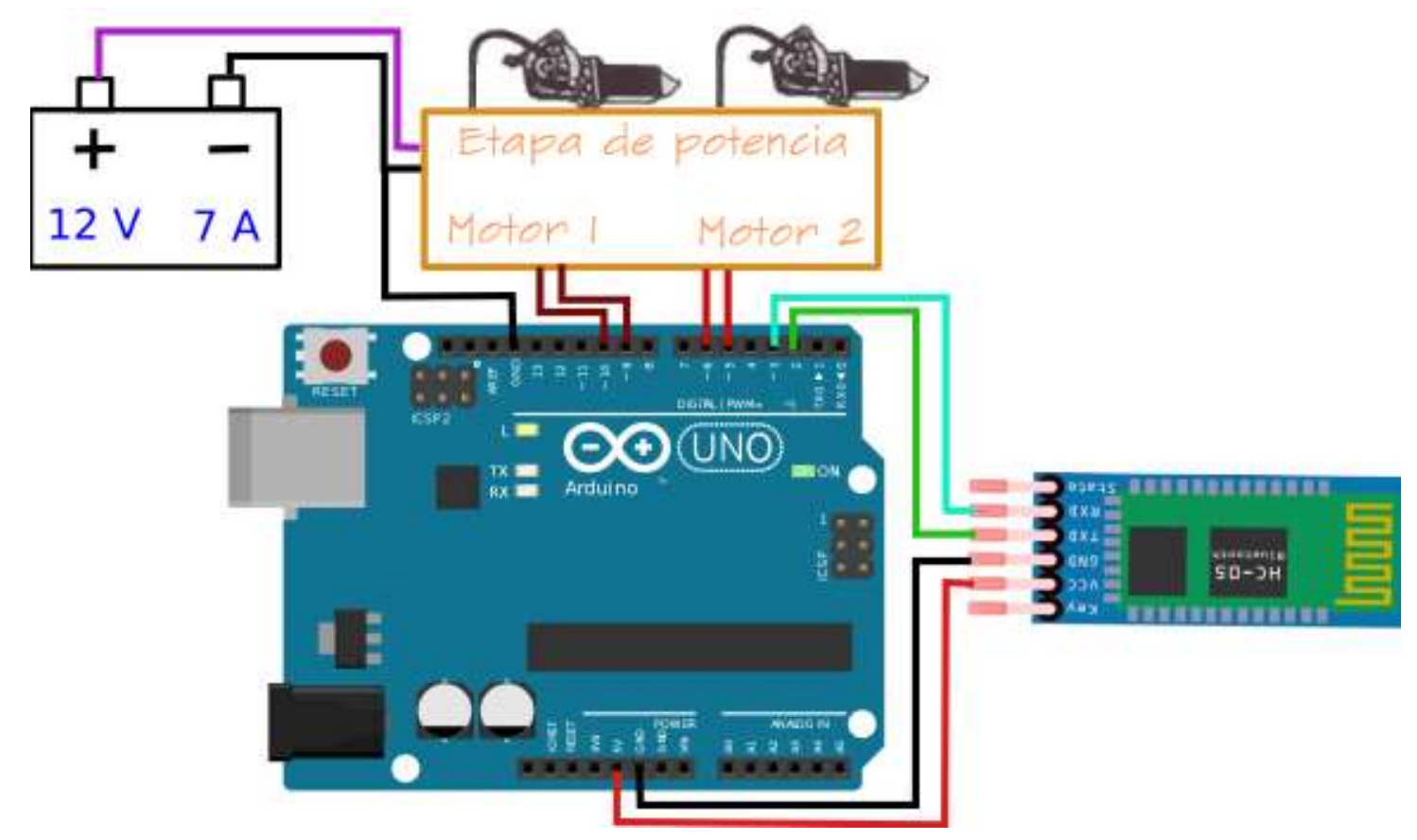

Fuente de consulta: Fuente propia

La programación de la etapa de control se realizó en el IDE de Arduino, un entorno de programación sencillo y potente para programar, pero además incluye las herramientas necesarias para compilar el programa y "quemar" el programa ya compilado en la memoria flash del microcontrolador. Además el IDE ofrece un sistema de gestión de librerías y placas muy práctico.

Un programa de Arduino se denomina sketch o proyecto y tiene la extensión .ino. Importante: para que funcione el sketch, el nombre del fichero debe estar en un directorio con el mismo nombre que el sketch. No es necesario que un sketch esté en un único fichero, pero si es imprescindible que todos los ficheros estén dentro del mismo directorio que el fichero principal. La estructura básica de un sketch de Arduino es bastante simple y se compone de al menos dos partes. Estas dos partes son obligatorios y encierran bloques que contienen declaraciones, estamentos o instrucciones.

- $\quad \operatorname{setup}()-\mathrm{http} / / /$ arduino.cc/en/Reference/Setup

- $\quad \operatorname{loop}()-\mathrm{http}: / /$ arduino.cc/en/Reference/Loop

Adicionalmente se puede incluir una introducción con los comentarios que describen el programa y la declaración de las variables y llamadas a librerías, Figura 8.15. setup() es la parte encargada de recoger la configuración y loop() es la que contiene el programa que se ejecuta cíclicamente (de ahí el término loop -bucle-). Ambas funciones son necesarias para que el programa trabaje. La estructura del sketch está definida en el siguiente enlace: http://arduino.cc/en/Tutorial/Sketch. 
Figura 8.15 Estructura de Sketch de IDE de Arduino

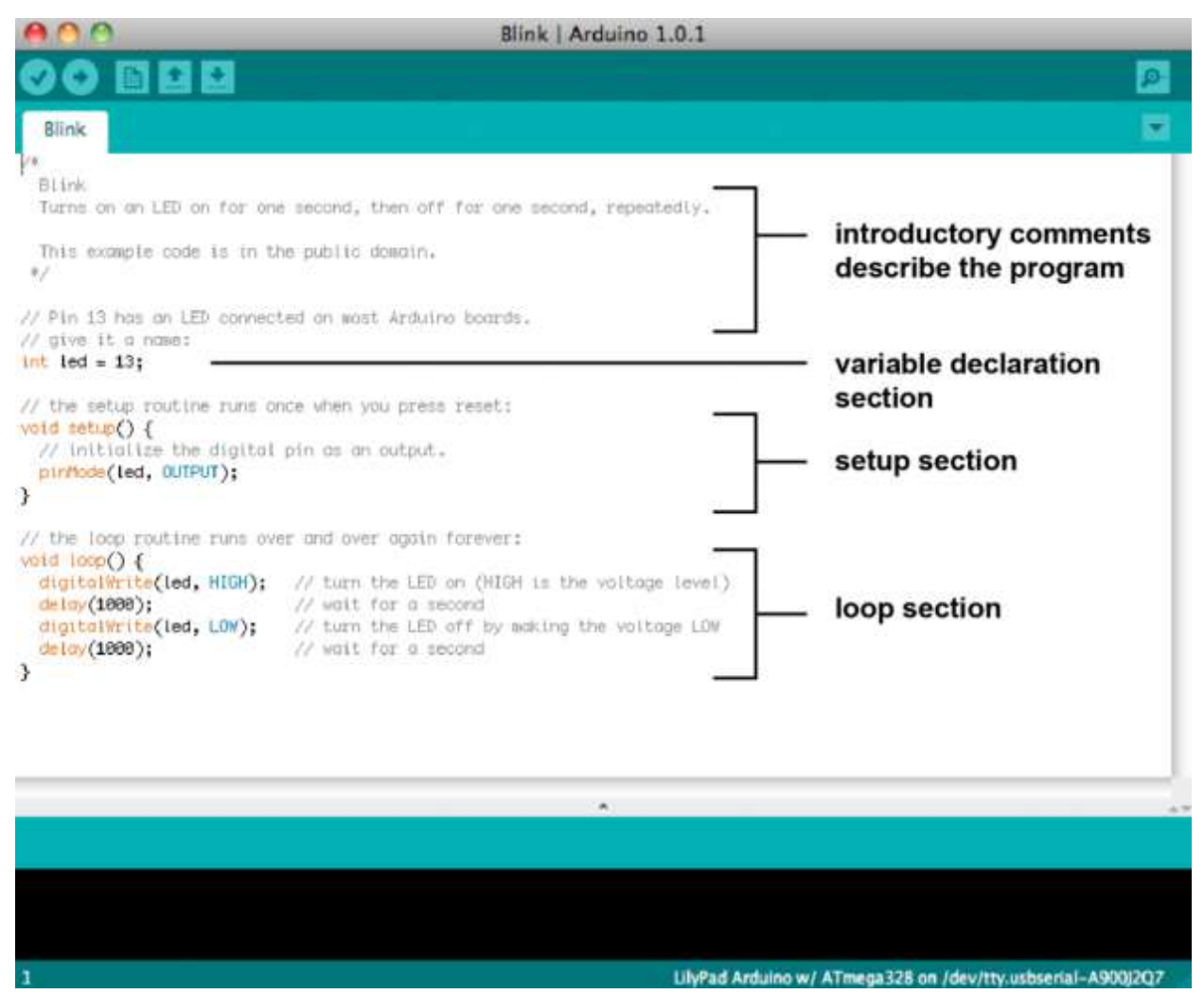

Fuente de consulta: https://aprendiendoarduino.wordpress.com/2017/01/23/programacion-arduino-5/

De manera símil al esquema de la Figura 8.1, la programación del sistema cuenta con dos etapas:

\section{Recepción e interpretación de datos del equipo Myo}

2. Asignación y envió de direccionamientos y velocidad a la etapa de potencia.

El diagrama de flujo de la primera etapa se puede observar en la Figura 8.16, para la programación de ésta se tomó de referencia el ejemplo PoseData de la librería MyoBrigde.

Figura 8.16 Diagrama de flujo de etapa de sensado electromiográfico

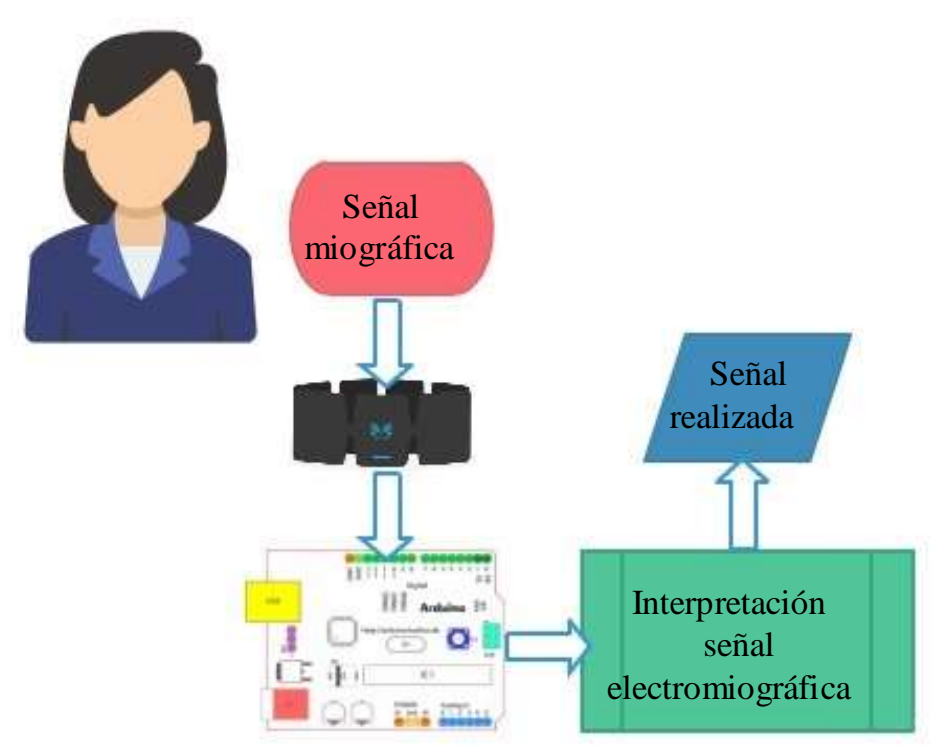

Fuente de consulta: Fuente propia

Para el funcionamiento de la segunda etapa fue necesario realizar una tabla de verdad de asignación de direccionamientos y velocidad acordes a los comandos recibidos de la Myo. 
Tabla 8.2 Asignación de direccionamiento y velocidades de acuerdo a señales electromiográficas

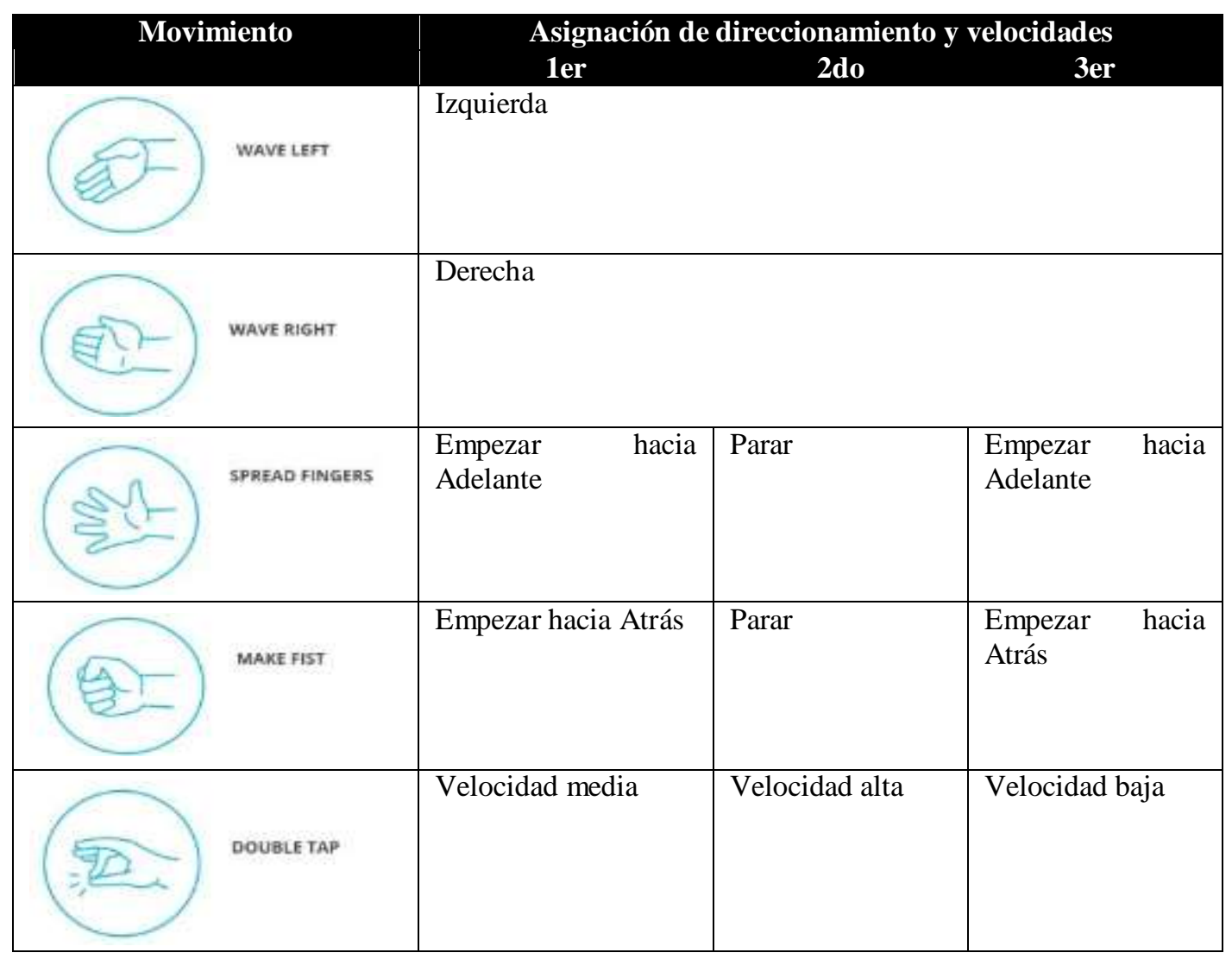

Fuente de consulta: Fuente propia

Con la definición de direcciones y velocidades el diagrama de flujo y tomando en cuenta ambas etapas queda como:

Figura 8.17 Diagrama de flujo de etapa de sensado electromiográfico y etapa de control

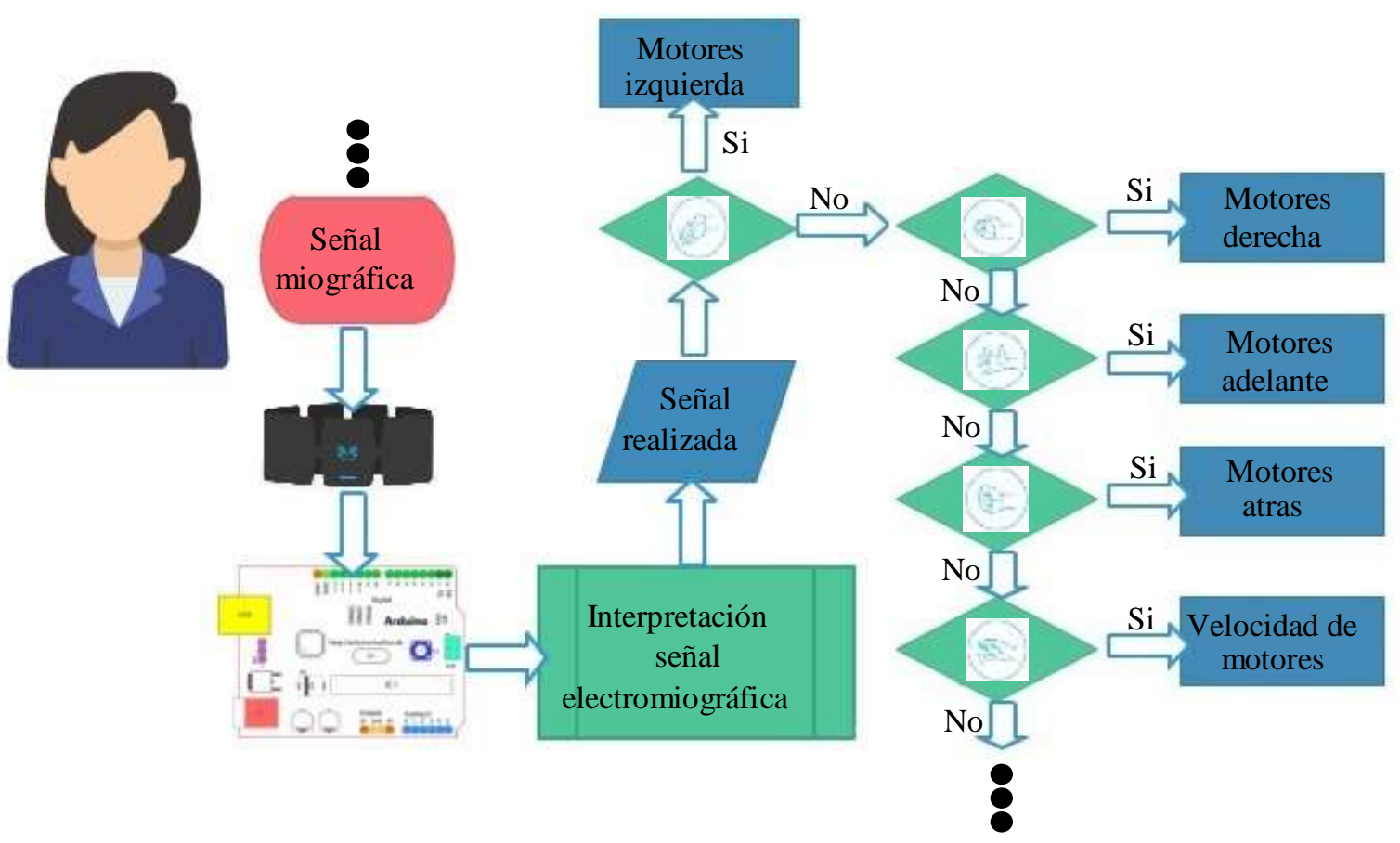

Fuente de consulta: Fuente propia

Como se puede apreciar en la tabla 8.2 algunas señas tienen dos o tres tiempos y en cada uno se realiza una acción diferente, esto se realiza en cada proceso de las señas mediante contadores. Estos procesos envían las señales pertinentes a la etapa de potencia para el movimiento deseado en los motores. 


\section{Etapa de acople mecánico}

El diseño e implementación del acople mecánico se realizó pensando en no realizar ninguna modificación a la silla de ruedas, para esto a los ejes de los motores se les acopló una llanta con rallado de manera fija, de esta manera al acercar los ejes de los motores al punto de hacer contacto con las ruedas de la silla será suficiente para moverla. En el diseño también se pensó en el soporte de los motores, la etapa de potencia, la etapa de control, así como de la batería de alimentación como se observa en la Figura 8.18.

Figura 8.18 Diseño de acople mecánico. (a) vista superior y (b) zoom de vista isométrica

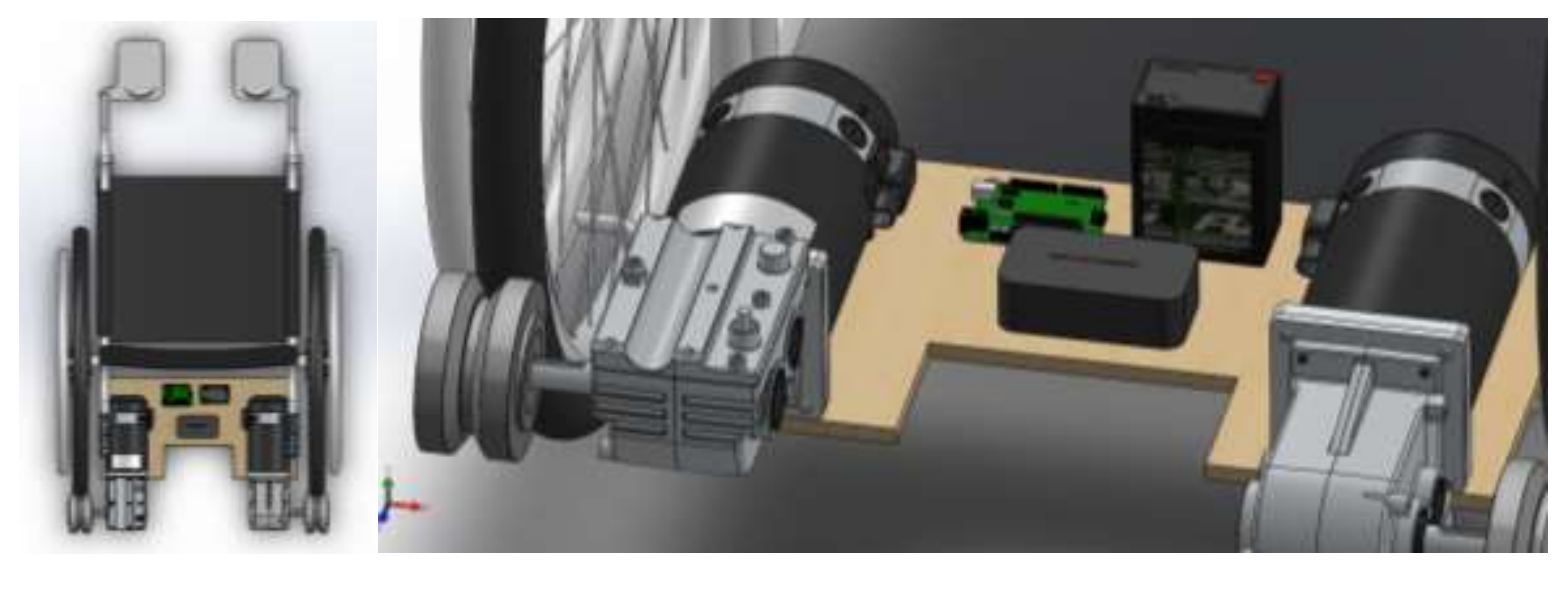

Fuente de consulta: Fuente propia

\section{Resultados}

El sistema propuesto cumplió con el objetivo deseado, facilitando la movilidad de una silla de ruedas mediante un control mioeléctrico. Los resultados específicos de cada una de las etapas del sistema de detallan a continuación.

\section{Etapa de sensado electromiográfico}

La interpretación de las señales mioleléctricas se verifico mediante una prueba donde se les pidió a cinco usuarios el siguiente procedimiento:

1. Crear y calibrar un usuario en el equipo Myo.

2. Realizar las señas solicitadas por medio de una pantalla de manera aleatoria.

3. Hacer cinco repeticiones del paso dos.

Los resultados de aciertos se pueden ver en la siguiente tabla:

Tabla 8.3 Resultados de aciertos de prueba de interpretación de señas mioeléctricas

\begin{tabular}{|l|l|l|l|l|l|l|}
\hline Usuario & \multicolumn{5}{|c|}{ Repetición } & Total \\
& $\mathbf{1}$ & $\mathbf{2}$ & $\mathbf{3}$ & $\mathbf{4}$ & $\mathbf{5}$ & \\
\hline $\mathbf{1}$ & $90 \%$ & $90 \%$ & $100 \%$ & $100 \%$ & $100 \%$ & $96 \%$ \\
\hline $\mathbf{2}$ & $80 \%$ & $100 \%$ & $100 \%$ & $100 \%$ & $100 \%$ & $96 \%$ \\
\hline $\mathbf{3}$ & $100 \%$ & $90 \%$ & $100 \%$ & $100 \%$ & $100 \%$ & $98 \%$ \\
\hline $\mathbf{4}$ & $90 \%$ & $100 \%$ & $100 \%$ & $100 \%$ & $100 \%$ & $98 \%$ \\
\hline $\mathbf{5}$ & $100 \%$ & $100 \%$ & $100 \%$ & $100 \%$ & $100 \%$ & $100 \%$ \\
\hline
\end{tabular}

Como se puede apreciar el sistema interpreta con $97.6 \%$ de eficiencia, sin embargo, el sensor mioeléctrico mejora su interpretación de acuerdo al número de repeticiones.

\section{Etapa de potencia}

La implementación de la etapa de potencia elaborada dio los resultados esperados en el control de los motores del sistema tanto en direccionamiento como en velocidad. Cabe destacar que las velocidades de los motores son adecuadas para la movilización de la silla de ruedas. 


\section{Etapa de control}

Por medio de esta etapa se logró un control adecuado de la silla de ruedas de acuerdo con la Tabla 8.2, los usuarios opinaron que el control es fácil de maniobrar y entender. De manera similar a la prueba del sensor mioeléctrico se realizaron pruebas de aciertos en los movimientos deseados en el sistema obteniendo un $98 \%$ de eficiencia en el sistema.

\section{Etapa de acople mecánico}

El acople mecánico diseñado fue el adecuado para la movilización de la silla de ruedas de acuerdo con los comandos señalados por la etapa de control, cabe destacar que es de fácil colocación y ajuste además de ser ligero.

\section{Agradecimientos}

Se agradece a la Universidad Tecnológica de Torreón por el apoyo brindado para el desarrollo de este trabajo y al cuerpo académico de innovación, integración y desarrollo de tecnologías.

\section{Conclusiones}

El sistema de control mioeléctrico propuesto obtuvo un $98 \%$ de eficiencia en la automatización de la silla de ruedas para los cinco usuarios que lo probaron, es de fácil implementación y asequible, cumpliendo así con el objetivo del sistema.

Cabe mencionar que la pila que se uso es recargable y el sistema tiene ser recargada cada tres horas si se encuentra en uso continuo, por lo que una oportunidad de mejora es implementar un sistema de alimentación que aporte más tiempo en uso. Otra cuestión es que el equipo Myo ya no se fabrica, aunque aún se vende, por lo que sería apropiado adecuar la etapa de control a otro tipo de sensores electromiográficos.

\section{Referencias}

Auat Cheein, F. A., De la Cruz, C., Carelli, R., \& Bastos Filho, T. F. (2011). Navegaci'on Aut'onoma Asistida Basada en SLAM. RIAI, 81 -92.

Villa Moreno, A., Gutiérrez Gutiérrez, E., \& Pérez Moreno, J. C. (2008). Consideraciones para el análisis de la marcha humana. Técnicas de videogrametría, electromiografía y dinamometría. Revista Ingeniería Biomédica, 16 -26.

Alonso Alonso, A., Hornero Sánchez, R., Espino Hurtado, P., De la Rosa Steinz, R., \& Liptak , L. (2002). Entrenador mioeléctrico de prótesis para amputados de brazo y mano . MAPFRE MEDICINA , $11-23$.

Bernal Moncivaiz, B. A., \& Martínez, N. N. (2019). Tipos de sillas de Ruedas. Estado de México: Universidad Autónoma del Estado de México.

Cano , R., Hincapie , J., \& Garcia , J. ( 2015). Especificación de una Adaptación Tecnológica a una Silla de Ruedas Eléctricas Aplicando un Enfoque Modular. Congreso Internacional sobre Innovación y Desarrollo Tecnológico (págs. 1 - 6 ). Cuernavaca, Morelos México.: ResearchGate.

Cano, R., Hincapie, J., \& Garcia M, J. (2014). CARACTERIZACIÓN DE REQUISITOS FUNCIONALES DE UNA SILLA DE RUEDAS ELÉCTRICA USANDO TÉCNICAS DEL DISEÑO DE PRODUCTOS CENTRADO EN EL USUARIO. Publicacion (págs. 1 - 8). colombia: ResearchGate.

D. Contreras, A., Ramírez-García, , F., \& Gallegos, I. , B. (2015). Prototipo de una Prótesis Mioeléctrica para. Revista de Ingenieria Biomédica, 77 -92 .

Daniel Lozano, 2. (2017). Arduino Práctico. . México: ANAYA. 
INEGI. (13 de Mayo de 2017). La discapacidad en México datos al 2014-2016. Obtenido de http://internet.contenidos.inegi.org.mx/contenidos/productos/prod_serv/contenidos/espanol/bvinegi/pro ductos/nueva_estruc/702825090203.pdf

Iñaki Aguirre, G., \& Balza, M. (2015). Diseño y construcción de un exoesqueleto de brazo con dos grados de libertad controlado con señales mioeléctricas para rehabilitación motora. Redalyc, 96 -105.

Ramírez, A., \& Garzón, D. (2008). Análisis de sensibilidad por la colocación de los electrodos en la electromiografía de superficie (semg). Redalyc, 70 - 79.

Salcedo Hernández, L. F., Torres San Miguel , C. R., Urriolagoitia Sosa , G., \& Romero, Á. B. (2011). Rediseño para la Optimización de una Silla de Ruedas. $10^{\circ}$ Congreso Nacional de Mecatrónica (págs. 90 - 96). Puerto Vallarta: Asociación Mexicana de Mecatrónica A.C. .

Torrente. (2015). Arduino. Curso práctico de formación,. México: Alfaomega Grupo Editor, S.A. de C.V.

Valero, J., Sandoval, C., Bonilla, Y., \& Duque, C. (2012). SISTEMA DE CONTROL DE UNA SILLA DE RUEDAS PARA SEGUIMIENTO AUTOMÁTICO. Memorias del Congreso ASME USB 2012: (págs. 1- 6). Caracas: ResearchGate. 